\title{
CARGAS SUPERFICIAIS DA FRAÇÃO ARGILA DE SOLOS INFLUENCIADAS PELA VINHAÇA E FÓSFORO
}

\author{
Bruno Teixeira Ribeiro* \\ Instituto Federal de Educação, Ciência e Tecnologia do Sul de Minas Gerais, Campus Machado, 37750-000 Machado - MG, Brasil \\ José Maria de Lima, Nilton Curi, Geraldo César de Oliveira e Pedro Luiz Terra Lima \\ Departamento de Ciência do Solo, Universidade Federal de Lavras, CP 37, 37200-000 Lavras - MG, Brasil
}

Recebido em 19/8/09; aceito em 28/7/10; publicado na web em 16/11/10

\begin{abstract}
SURFACE CHARGE OF CLAY FRACTION AS AFFECTED BY VINASSE AND PHOSPHORUS. Effects of vinasse, P sorption and the interaction vinasse-phosphorus on zeta potential and point of zero charge (PZC) as well as the effects of vinasse on P sorption on clay-fraction samples from two soils were evaluated. The vinasse and P sorption influenced the surface charge of clay fraction of both soils. Sorption of P increased negative charges from soil particles reducing PZC. These effects were more pronounced when clay fraction was previously treated with vinasse. Vinasse treatment reduced P sorption, probably due to coating of P-adsorption sites and by enhancing the negative charges.
\end{abstract}

Keywords: zeta potential; point of zero charge (PZC); soil charges.

\section{INTRODUÇÃO}

As cargas na superfície das partículas dos solos estão relacionadas aos fenômenos de superfície, como a dispersão/floculação, ${ }^{1,2}$ capacidade de troca de cátions e de ânions, ${ }^{3}$ adsorção de pesticidas ${ }^{4}$ e de metais pesados. ${ }^{5}$ As cargas do solo podem ser do tipo permanente, quando originadas de substituição isomórfica na formação dos minerais, principalmente da fração argila, ou dependentes do $\mathrm{pH}$ do meio (variáveis), ${ }^{6,7}$ sendo essas últimas comuns em solos tropicais altamente intemperizados ricos em óxidos de ferro e de alumínio. ${ }^{8}$ As cargas variáveis representam mais de $70 \%$ da carga total em amostras da superfície de Latossolos. ${ }^{9}$

Uma das formas de avaliar as cargas superficiais dos coloides do solo é por meio do potencial zeta $(\zeta)$, definido como a carga elétrica líquida que se desenvolve no plano que divide a camada de Stern e a camada difusa a um determinado $\mathrm{pH} .{ }^{6} \mathrm{O} \zeta$ é influenciado principalmente pela concentração eletrolítica e pelo $\mathrm{pH}$ da suspensão coloidal. ${ }^{6,10,11}$ Quando o $\zeta$ é igual a zero, o ponto de carga zero (PCZ) da partícula coloidal é alcançado. ${ }^{6}$

A carga elétrica e o PCZ são dependentes da proporção dos constituintes minerais e orgânicos do solo. Os óxidos de ferro e de alumínio contribuem para o aumento das cargas positivas e do PCZ, enquanto a matéria orgânica e argilominerais silicatados, como a caulinita, aumentam as cargas negativas e reduzem o PCZ.,12-14 Além disso, a introdução de cátions, ânions e moléculas no meio podem afetar reações de protonação/desprotonação, influenciando as cargas superficiais e o PCZ dos coloides do solo..$^{15-17}$

Solos ricos em óxidos de ferro e de alumínio apresentam elevada adsorção de $\mathrm{P},{ }^{18-20}$ exigindo adubações pesadas desse nutriente para se ter uma disponibilidade adequada para as plantas. Além de sua elevada adsorção, o P causa significativas mudanças nas cargas superficiais das partículas do solo, tornando-as mais eletronegativas e reduzindo o PCZ. ${ }^{16,17,21}$ Tais alterações, além dos benefícios da adubação fosfatada, podem contribuir para o aumento da capacidade de troca de cátions no solo, ${ }^{3}$ importante no manejo da fertilidade do solo e, também, influenciar o comportamento sortivo de metais pesados e pesticidas. ${ }^{22,23}$ Por outro lado, o aumento da

*e-mail: ribeiro.bt@gmail.com carga líquida negativa dos coloides pode facilitar a dispersão das partículas do solo. ${ }^{16}$

Em razão da elevada adsorção de $\mathrm{P}$, principalmente em solos oxídicos, algumas práticas de manejo do solo têm sido adotadas no sentido de reduzir sua adsorção e aumentar sua disponibilidade para as plantas. Entre elas, destaca-se a adição de matéria orgânica ao solo, uma vez que os compostos orgânicos podem competir pelos mesmos sítios de adsorção de $\mathrm{P}^{24-26}$

A vinhaça, principal resíduo de usinas de processamento de cana-de-açúcar, foi por muito tempo lançada em cursos d'água como forma de descarte. Atualmente, tal prática é proibida por lei; uma das alternativas de seu uso é a aplicação em solos como fertilizante. Alguns efeitos da vinhaça no solo são bem conhecidos, tais como alterações no $\mathrm{pH}$, aumento no carbono orgânico, capacidade de troca de cátions, atividade microbiológica e melhoria de alguns atributos físicos. ${ }^{27-33}$ Entretanto, ainda é desconhecido o efeito da vinhaça nas cargas superficiais e no ponto de carga zero (PCZ) em solos, sobretudo associado a outras práticas de manejo, como a adubação fosfatada, considerando a importância desta em solos tropicais altamente intemperizados.

Conduziu-se este trabalho com base na hipótese de que compostos orgânicos presentes na vinhaça, uma vez adsorvidos nas partículas do solo, podem alterar as cargas superficiais e ainda competir por sítios de adsorção de $\mathrm{P}$, podendo aumentar sua disponibilidade na solução do solo. Nesse contexto, ressalta-se que a adsorção de compostos orgânicos foi maior nas amostras dos horizontes B que nas dos horizontes A, pois as amostras dos horizontes B possuem maiores teores de óxidos de ferro e argilominerais e as amostras do horizonte A têm mais matéria orgânica. ${ }^{34} \mathrm{~A}$ maior capacidade de adsorção dos óxidos é atribuída aos seus maiores valores de PCZ, fazendo com que esses materiais desenvolvam cargas positivas, como exemplo, em condições de $\mathrm{pH}$ da maioria dos solos cultivados (5-6), favorecendo a adsorção dos compostos orgânicos negativamente carregados.

Os objetivos deste trabalho foram avaliar o efeito da vinhaça, da adsorção de fósforo e a interação vinhaça-fósforo sobre o potencial zeta e o ponto de carga zero em amostras da fração argila de dois solos de mineralogia contrastante quanto aos teores de ferro e, também, o efeito da vinhaça na adsorção de $\mathrm{P}$ nesses materiais. 


\section{PARTE EXPERIMENTAL}

\section{Caracterização dos solos utilizados}

Foram escolhidos dois solos de mineralogia contrastante quanto aos teores de ferro, sendo utilizadas amostras da fração argila do horizonte B de um Latossolo Vermelho Distroférrico (LVdf), (camada de $70-90 \mathrm{~cm}$ de profundidade), localizado em Santo Ângelo, RS, $28^{\circ} 30^{\prime} \mathrm{S}$ e $54^{\circ} 27^{\prime} \mathrm{W}$, textura muito argilosa, originado de basalto, com predominância de caulinita e hematita na fração $\operatorname{argila~}^{35} \mathrm{e} \mathrm{um}$ Gleissolo Melânico (camada de $65-90 \mathrm{~cm}$ de profundidade), localizado no Planalto Central brasileiro (19 $30^{\circ}$ 'S e $48^{\circ} 08^{\prime} \mathrm{W}$ ), textura argilosa, que ocorre ao longo da linha de drenagem da chapada, com teor de gibbsita superior a $950 \mathrm{~g} \mathrm{~kg}^{-1}$ na fração argila, sendo esse material referenciado como gibbsita pedogenética, ${ }^{36}$ ou simplesmente gibbsita. Foi realizada a caracterização do complexo sortivo dos solos utilizados, conforme Embrapa. ${ }^{37}$

Para obtenção da fração argila, $50 \mathrm{~g}$ de terra fina seca ao ar (TFSA) foram colocados em aproximadamente $250 \mathrm{~mL}$ de água destilada $+10 \mathrm{~mL}$ de $\mathrm{NaOH} 0,1 \mathrm{~mol} \mathrm{~L}^{-1}$ e dispersos mecanicamente em agitador tipo coqueteleira por $20 \mathrm{~min}$. Após esse período, a fração areia foi removida por peneiramento. A suspensão (silte + argila) foi transferida para proveta com capacidade de $1.000 \mathrm{~mL}$, obtendo-se a fração argila por sifonamento após o tempo de sedimentação da fração silte, conforme Embrapa. ${ }^{37}$

Os materiais foram caracterizados por difratometria de raios-X (método do pó), utilizando um aparelho Philips, com variação angular de $4-50^{\circ}(2 \theta)$, radiação de $\operatorname{CuK} \alpha(\lambda=1,54051 \AA$ ) e velocidade de exposição de $1^{\circ} \theta \min ^{-1}$.

\section{Vinhaça}

A vinhaça foi obtida em um alambique de cachaça artesanal apresentando como principais características: $\mathrm{pH}(3,5)$; $\mathrm{CE}$ (7,5 dS $\left.\mathrm{m}^{-1}\right) ; \mathrm{C}_{\text {org }}\left(9,6 \mathrm{~g} \mathrm{~L}^{-1}\right) ; \mathrm{K}^{+}\left(2,5 \mathrm{~g} \mathrm{~L}^{-1}\right)$. O teor de carbono orgânico foi determinado por meio da digestão de $0,5 \mathrm{~mL}$ da vinhaça e quantificado pela titulação com sulfato ferroso amoniacal. ${ }^{38} \mathrm{O} \mathrm{K}^{+}$foi determinado diretamente, realizando-se as diluições necessárias, por meio de fotômetro de chama.

\section{Tratamento da fração argila dos solos com vinhaça}

Em frascos de vidro com capacidade para $20 \mathrm{~mL}$, foram adicionados $0,1 \mathrm{~g}$ da fração argila e $10 \mathrm{~mL}$ de vinhaça (relação $1: 100$ ) procedendo-se, então, à agitação por $4 \mathrm{~h}^{34}$ à temperatura ambiente (22 ${ }^{\circ} \mathrm{C} \pm 2$ ), em agitador do tipo horizontal (130 rpm). Após a agitação, as amostras foram centrifugadas por $10 \mathrm{~min}$ a $500 \mathrm{~g}$, realizando-se as leituras de $\mathrm{pH}$ e $\mathrm{CE}$ do sobrenadante, sendo esse descartado e a fração argila congelada e liofilizada.

\section{Ensaio de adsorção de $P$}

Amostras da fração argila tratadas e não tratadas com vinhaça foram submetidas ao ensaio de adsorção de $\mathrm{P},{ }^{16}$ com modificações. Para isso, $10 \mathrm{~mL}$ de solução de diferentes concentrações iniciais de $\mathrm{P}\left(0 ; 0,08 ; 0,16 ; 0,32 ; 0,64 ; 1,28 \mathrm{e} 2,56 \mathrm{mmol} \mathrm{L}^{-1}\right)$, tendo como fonte $\mathrm{KH}_{2} \mathrm{PO}_{4}$ (P.A) e preparadas em $\mathrm{NaCl} 0,01 \mathrm{~mol} \mathrm{~L}^{-1}(\mathrm{pH}=5,5 \pm 0,02)$ como solução de fundo, foram adicionadas a $0,1 \mathrm{~g}$ da fração argila em frascos com capacidade para $20 \mathrm{~mL}$ e mantidas em agitação (agitador horizontal - $130 \mathrm{rpm}$ ) por $72 \mathrm{~h}$, alternando-se $12 \mathrm{~h}$ de agitação e $12 \mathrm{~h}$ de repouso, em condições normais de temperatura e pressão. As amostras com vinhaça foram mantidas nos mesmos frascos onde foi realizado o tratamento. Após o período de agitação, as amostras foram centrifugadas por $10 \mathrm{~min}$ a $500 \mathrm{~g}$ e o sobrenadante foi recolhido para determinação de P. As amostras foram congeladas, liofilizadas e armazenadas em dessecador para realização das análises para avaliação do potencial zeta. A determinação de $\mathrm{P}$ foi realizada por colorimetria, conforme Embrapa. ${ }^{37}$ A quantidade de $\mathrm{P}$ adsorvida foi calculada pela diferença entre a concentração inicial e final de $\mathrm{P}$ na solução (no sobrenadante) e expressa em mmol kg-1 da fração argila:

$$
\mathrm{P} \text { adsorvido }=\left(\mathrm{P}_{\text {inicial }}-\mathrm{P}_{\text {final }}\right) . \mathrm{V} / \mathrm{m}
$$

em que $\mathrm{P}_{\text {inicial }}$ é a concentração de $\mathrm{P}$ na solução inicial; $\mathrm{P}_{\text {final }}$ é a concentração de $\mathrm{P}$ no sobrenadante; $\mathrm{V}$ é o volume de solução $(10 \mathrm{~mL})$ e $\mathrm{m}$ é a massa da fração argila utilizada em $\mathrm{kg}$.

Os resultados da adsorção de $\mathrm{P}$ foram ajustados ao modelo de Langmuir, utilizando planilhas eletrônicas, disponíveis para download (ars.usda.gov/msa/awmru/bolster/Sorption_spreadsheets), de acordo com Bolster e Hornberger. ${ }^{39}$

\section{Determinação do potencial zeta $(\zeta)$}

A determinação do potencial zeta foi realizada de acordo com a metodologia descrita em Lima et al. ${ }^{16}$ com modificações. Os materiais de argila presentes nos frascos (tratados ou não tratados previamente com vinhaça e P) foram suspensos em $40 \mathrm{~mL}$ de solução de $\mathrm{NaCl}$ $0,01 \mathrm{~mol} \mathrm{~L}^{-1}$. Dessa suspensão, foram pipetados $4 \mathrm{~mL}$, transferindo-se para béquer de $50 \mathrm{~mL}$ contendo outros $40 \mathrm{~mL}$ de solução de $\mathrm{NaCl}$ $0,01 \mathrm{~mol} \mathrm{~L}^{-1}$ em diferentes valores de $\mathrm{pH}(2,3,5,6,7,9$ e $11 \pm 0,2)$, ajustados previamente por meio de $\mathrm{HCl}$ ou $\mathrm{NaOH} 0,01 \mathrm{M}$. Essa suspensão de argila, correspondendo a aproximadamente $250 \mathrm{mg}$ $\mathrm{L}^{-1}$, foi transferida para célula eletroforética do aparelho Zeta Meter $3.0+$, procedendo-se às leituras de potencial zeta. Todas as análises foram realizadas em triplicata, com 10 a 20 leituras por repetição.

\section{Tratamento estatístico}

Os resultados foram submetidos à análise de variância e os valores de potencial zeta foram plotados em função do $\mathrm{pH}$, sendo ajustados modelos polinomiais cúbicos, utilizando o software Sigma Plot 10.0. ${ }^{40}$ Com base nesses modelos, foi estimado o $\mathrm{pH}$ em que o potencial zeta se iguala a zero (considerando-se duas casas decimais - 0,00), correspondendo ao ponto de carga zero (PCZ). Os valores de PCZ foram relacionados com as quantidades de $\mathrm{P}$ adsorvidas na fração argila, na ausência e presença da vinhaça.

\section{RESULTADOS E DISCUSSÃO}

Efeito da vinhaça, da adsorção de $P$ e da interação vinhaça-P sobre o potencial zeta $(\zeta)$ e ponto de carga zero (PCZ)

A fração argila dos solos apresentou carga superficial dependente do $\mathrm{pH}$ (Figura 1). Os modelos polinomiais cúbicos ajustados foram altamente significativos $(\mathrm{P}<0,0001)$, com valores de $\mathrm{R}^{2}$ variando de 0,94 a 0,99 . O potencial zeta da amostra controle do LVdf (sem tratamento com vinhaça e P) variou de $+20 \mathrm{mV}$ em pH 2,0 até -19 $\mathrm{mV}$ em pH 11,0, com o PCZ (potencial zeta igual a zero) em $\mathrm{pH}$ 7,5 (Figura 1). A gibbsita mostrou-se mais positivamente carregada, apresentando um potencial zeta de $+56 \mathrm{mV}$ em pH 2,0, apresentando $-12 \mathrm{mV}$ em pH próximo de 11,0, com o PCZ em 9,2. Hou et al. ${ }^{41}$ também observaram que a gibbsita se apresentou mais positivamente carregada, comparativamente aos óxidos de ferro, porém, em materiais sintéticos. Os valores de PCZ encontrados neste trabalho para o LVdf e gibbsita corroboram aqueles propostos para materiais ricos em óxidos de ferro e de alumínio, que variam entre 7,8 e 9,5. ${ }^{7}$ 


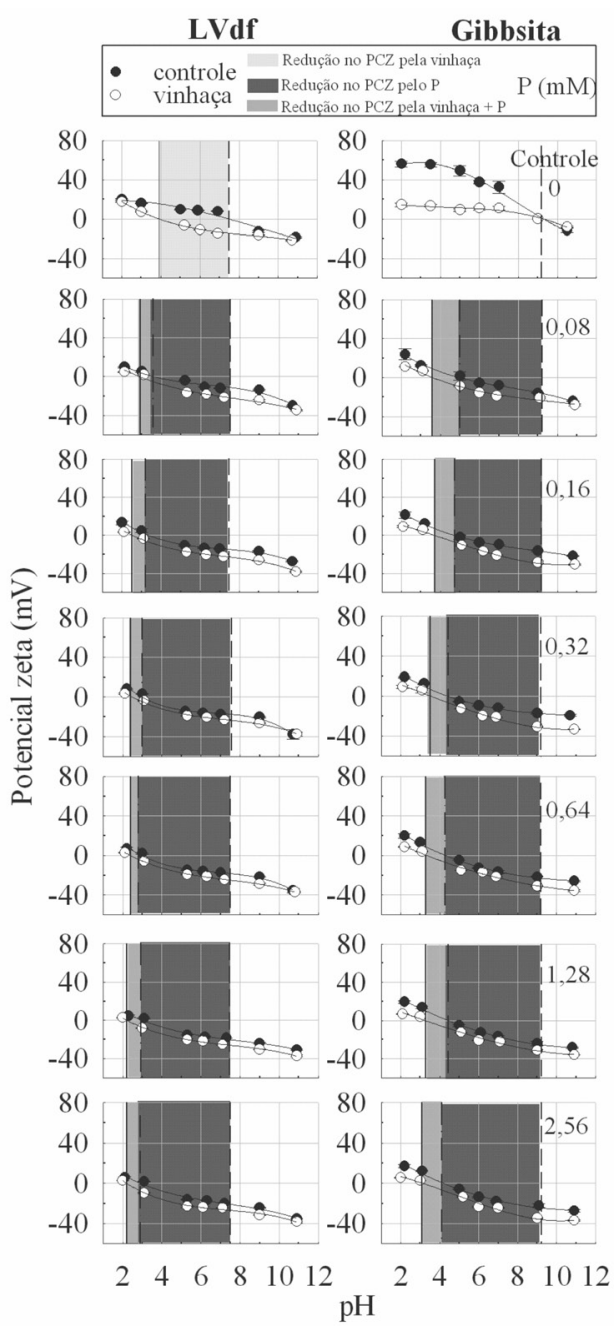

Figura 1. Efeito da vinhaça e da concentração inicial de P sobre o potencial zeta e ponto de carga zero (PCZ) da fração argila do Latossolo Vermelho Distroférrico ( LVdf) e gibbsita. Barras de erro verticais indicam o desvio-padrão da média (n=3). Linhas verticais: tracejadas indicam o PCZ controle do LVdf e gibbsita; tracejadas-pontilhadas, o PCZ desses materiais após tratamento com P e contínuas, após o tratamento com vinhaça elou vinhaça + fósforo

Giaveno et al. ${ }^{21}$ avaliaram o potencial zeta de hematita e gibbsita sintéticas e da fração argila de dois Latossolos do Mato Grosso do Sul sob diferentes ambientes, Cerrado nativo e pastagem. Para hematita, o potencial zeta variou de $+30 \mathrm{mV}$ em $\mathrm{pH}$ próximo de 3,0 até $-20 \mathrm{mV}$ em $\mathrm{pH}$ 11,0, alcançando o PCZ em pH 7,0. A gibbsita, nessa mesma faixa de $\mathrm{pH}$, variou o potencial zeta de +37 até $-20 \mathrm{mV}$, aproximadamente, alcançando o PCZ em 5,8. A fração argila do Latossolo sob Cerrado nativo teve seu potencial zeta variando de $+8 \mathrm{mV}(\mathrm{pH} 2,5)$ até $-30 \mathrm{mV}$ em $\mathrm{pH}$ próximo de 7,0, alcançando o $\mathrm{PCZ}$ em pH 3,9. No Latossolo sob pastagem, a fração argila apresentou mais carga positiva em $\mathrm{pH}$ 2,5 (+18 mV), sendo o PCZ alcançado em pH 5,3, tornando-se, a partir desse valor, negativa e alcançando $-25 \mathrm{mV}$ em $\mathrm{pH} 7,0$. A maior eletronegatividade e menor PCZ do Latossolo sob Cerrado nativo foram atribuídos ao maior conteúdo de matéria orgânica.

A maior quantidade de cargas positivas e o maior PCZ da gibbsita, comparativamente à fração argila do LVdf, podem estar relacionados com o conteúdo de carbono orgânico (4 vezes menor na gibbsita) (Tabela 1S, material suplementar), o qual contribui para cargas negativas e redução do $\mathrm{PCZ}^{3} \mathrm{e}$, também, pelo fato de esse material ser constituído quase que exclusivamente por óxido de alumínio (> 950 $\mathrm{g} \mathrm{kg}^{-1}$ na fração argila), ${ }^{36,42}$ que contribui para aumentar as cargas positivas e o PCZ. No LVdf, além dos óxidos de ferro e de alumínio, há a presença de caulinita (Figura $1 \mathrm{~S}$, material suplementar) e maiores teores de $\mathrm{SiO}_{2}$ (Tabela 1S), que reduzem o PCZ do material. ${ }^{40}$

A fração argila do LVdf tratada com vinhaça (Figura 1) apresentou valores de potencial zeta bem próximos da amostra controle quando em pH 2,0 e 11,0. Entretanto, observa-se que o PCZ da fração argila tratada com vinhaça foi alcançado em $\mathrm{pH} 3,9$, apresentando uma carga líquida negativa acima desse valor. Para a gibbsita, a vinhaça não alterou o PCZ; no entanto, na faixa de $\mathrm{pH}$ 2,0 a 9,2, se observa uma redução significativa do potencial zeta, sem, contudo, apresentar uma carga líquida negativa.

As cargas superficiais e o PCZ dependem da proporção de cada componente de carga variável presente no sistema, e a introdução de moléculas que são adsorvidas sobre as superfícies minerais é capaz de modificar reações de protonação/desprotonação., ${ }^{3,17}$ Dessa forma, compostos orgânicos (negativamente carregados e de baixo PCZ), como os presentes na vinhaça, ${ }^{33}$ uma vez adsorvidos na fração argila, contribuíram para o aumento das cargas negativas e a redução do PCZ dos materiais. É estimado para solos que o valor do PCZ pode ser reduzido em até uma unidade para cada aumento de $1 \%$ na matéria orgânica. ${ }^{3,14}$ Em amostras de três Latossolos mineralogicamente semelhantes, mas com diferentes proporções de caulinita, hematita, goethita e gibbsita, a remoção sequencial da matéria orgânica levou a um aumento do PCZ. ${ }^{14}$ Dobbss et al. ${ }^{43}$ observaram que a extração de ácidos húmicos e fúlvicos de amostras superficiais de Latossolos foi capaz de inverter o sinal da carga de superfície (de negativo para positivo) e aumentar o ponto de efeito salino nulo (PESN) - valor de $\mathrm{pH}$ em que a quantidade cargas positivas é igual à quantidade de cargas negativas independentemente da concentração salina do meio. Esse efeito da remoção da matéria orgânica foi mais destacado nos Latossolos de natureza gibbsítica, comparativamente aos cauliníticos.

Apesar dos efeitos observados sobre o potencial zeta e PCZ pelo tratamento da fração argila dos solos com vinhaça (Figura 1), tais alterações podem ser apenas temporárias, a menos que sucessivas aplicações de vinhaça sejam feitas, como de fato tem acontecido em áreas de usinas de álcool e alambique que aplicam a vinhaça como fertilizante, uma vez que os compostos orgânicos presentes na vinhaça são facilmente decomponíveis. Foi encontrado que apenas $12,8 \%$ do total de carbono orgânico solúvel presente na vinhaça permaneceram após 28 dias de incubação em condições aeróbicas. ${ }^{33}$ Aliado a isso, um maior efeito residual da vinhaça nas alterações das cargas superficiais de solos será esperado naqueles de natureza mais oxídica, partindo do pressuposto da maior adsorção e energia de ligação dos compostos orgânicos com os óxidos comparativamente aos argilominerais, como a caulinita. ${ }^{34}$

A adsorção de fósforo afetou o potencial zeta da fração argila dos solos (Figura 1), corroborando estudos prévios, nos quais se verificou que a adsorção específica do ânion fosfato torna a superfície carregada mais negativamente e também reduz o PCZ. ${ }^{16,17,20,21} \mathrm{~A}$ adsorção de $\mathrm{P}$ tornou a superfície da fração argila de ambos os solos mais eletronegativa e reduziu seu PCZ, sendo essas alterações mais pronunciadas quando as amostras foram previamente tratadas com vinhaça. Para o LVdf, sem vinhaça, na menor concentração inicial de $\mathrm{P}(0,08 \mathrm{mM})$, observa-se uma redução do potencial zeta, alcançando o PCZ em 3,6 e apresentando uma carga líquida negativa acima desse valor. Com o aumento da concentração inicial de P, observa-se uma leve redução do potencial zeta, atingindo-se o PCZ de 2,9 na maior concentração inicial de $\mathrm{P}(2,56 \mathrm{mM})$. Na presença da vinhaça, já com a concentração inicial de $\mathrm{P}(0,08 \mathrm{mM})$, atinge-se o $\mathrm{PCZ}$ de 2,9 , atingindo 2,2 na maior concentração inicial de P. Tendência semelhante foi observada para a gibbsita. Na menor concentração inicial de $\mathrm{P}$, o PCZ equivale a aproximadamente 5,0 e 3,6, na ausência e presença da vinhaça, respectivamente. Na maior concentração inicial de $\mathrm{P}$, 
foram obtidos valores de PCZ de 4,2 e 3,1 (na ausência e presença da vinhaça, respectivamente).

Giaveno et al. ${ }^{21}$ também avaliaram o efeito da adsorção de $\mathrm{P}$ no potencial zeta, em condições de $\mathrm{pH} 4,5$, da fração argila de Latossolos, hematita e gibbsita sintéticas descritos anteriormente. A fração argila dos Latossolos, com potencial zeta inicial entre 0 e $+10 \mathrm{mV}$ (controle), tornou-se eletronegativa com a presença de $\mathrm{P}$, mesmo na menor adição inicial de $\mathrm{P}(0,032 \mathrm{mM})$, alcançando $-22 \mathrm{mV}$ na maior adição inicial de $\mathrm{P}(1,61 \mathrm{mM})$. Hematita inicialmente apresentou potencial zeta de $+20 \mathrm{mV}$, permanecendo positivamente carregada até a adição inicial de $\mathrm{P}$ de $0,16 \mathrm{mM}$, tornando-se negativa $(-20 \mathrm{mV})$ com a dose de 0,32 mM, alcançando $-25 \mathrm{mV}$ na maior dose de P. Gibbsita, inicialmente com potencial de $+16 \mathrm{mV}$, tornou-se negativa $(-2,9 \mathrm{mV})$ com a adição inicial de $\mathrm{P}$ de $0,064 \mathrm{mM}$, alcançando $-13 \mathrm{mV}$ na maior dose $(1,61 \mathrm{mM})$. Considerando-se a mesma condição de $\mathrm{pH}(4,5)$, os resultados aqui encontrados foram semelhantes aos obtidos por Giaveno et al.. ${ }^{21} \mathrm{O}$ LVdf inicialmente (controle) apresentou potencial zeta de aproximadamente $+13 \mathrm{mV}$, tornando-se negativo $(-4 \mathrm{mV})$ com a dose inicial de $\mathrm{P}$ utilizada $(0,08 \mathrm{mM})$, alcançando $-11 \mathrm{mV}$ com a maior dose (2,56 mM) (Figura 1). Para gibbsita, inicialmente com potencial zeta $+51 \mathrm{mV}$, com a menor dose de $\mathrm{P}$ atingiu um potencial zeta de $+2 \mathrm{mV}$, tornando-se negativa com aumento das doses de $\mathrm{P}$, alcançando $-2 \mathrm{mV}$ na maior dose.

\section{Relação entre a quantidade adsorvida de P e o PCZ}

Apesar de o P ter exercido grande influência na redução do potencial zeta e do PCZ, tornando a superfície mais eletronegativa, o efeito de concentração parece ser pouco expressivo, pelo menos até o limite empregado no presente estudo. Na Figura 2 é apresentada a relação entre a quantidade de P adsorvida e o PCZ, ajustada a um modelo exponencial 3-parâmetros, com valores de $\mathrm{R}^{2}$ variando de 0,97 a 0,99.

Observa-se pela Figura 2 que já na menor quantidade adsorvida de $\mathrm{P}$ (aproximadamente 7,0 $\mathrm{mmol} \mathrm{kg}^{-1}$ para ambos materiais) tem-se uma redução expressiva do $\mathrm{PCZ}$, atingindo um patamar caracterizado por uma leve redução do PCZ com o aumento da quantidade adsorvida.

$\mathrm{O}$ efeito do $\mathrm{P}$ foi mais pronunciado na presença da vinhaça em ambos os materiais (Figura 2). No LVdf, o tratamento da fração argila apenas com vinhaça foi equivalente ao efeito da adsorção de $\mathrm{P}$ de

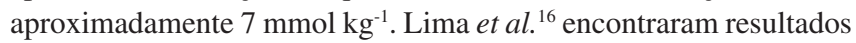
semelhantes para o efeito do $\mathrm{P}$ residual (após dessorção) na redução do ponto isoelétrico no horizonte B de Latossolos, não sendo encontradas diferenças para o horizonte A (mais rico em matéria orgânica), o que foi atribuído ao deslocamento de compostos orgânicos pelo $\mathrm{P}$, o que parece não ter ocorrido no presente trabalho.

$\mathrm{O}$ efeito da adsorção de $\mathrm{P}$ na redução do $\mathrm{PCZ}$, em relação às amostras-controle, foi mais pronunciado para o LVdf (Figura 3). Observa-se que para esse material, na menor quantidade de $\mathrm{P}$ adsorvida, tem-se uma redução de $52 \%$ no PCZ, enquanto para gibbsita tem-se uma redução de $47 \%$. Nas maiores quantidades adsorvidas, reduções de 61 e 55\% foram observadas para o LVdf e gibbsita, respectivamente. Na presença da vinhaça, para a menor quantidade de $\mathrm{P}$ adsorvida, tanto o LVdf quanto a gibbsita tiveram o valor de PCZ reduzido em $61 \%$ em relação ao controle. No entanto, com o aumento da adsorção de $\mathrm{P}$, o LVdf também teve uma maior redução relativa do PCZ, atingindo $70 \%$, e a gibbsita, $66 \%$ na condição de máxima adsorção de P.

\section{Efeito da vinhaça na adsorção de $\mathbf{P}$}

Em todas as soluções iniciais de P, a fração argila do LVdf e a gibbsita não diferiram significativamente quanto às quantidades adsorvidas de $\mathrm{P}$ e à capacidade máxima de adsorção (CMAP) (Fi-
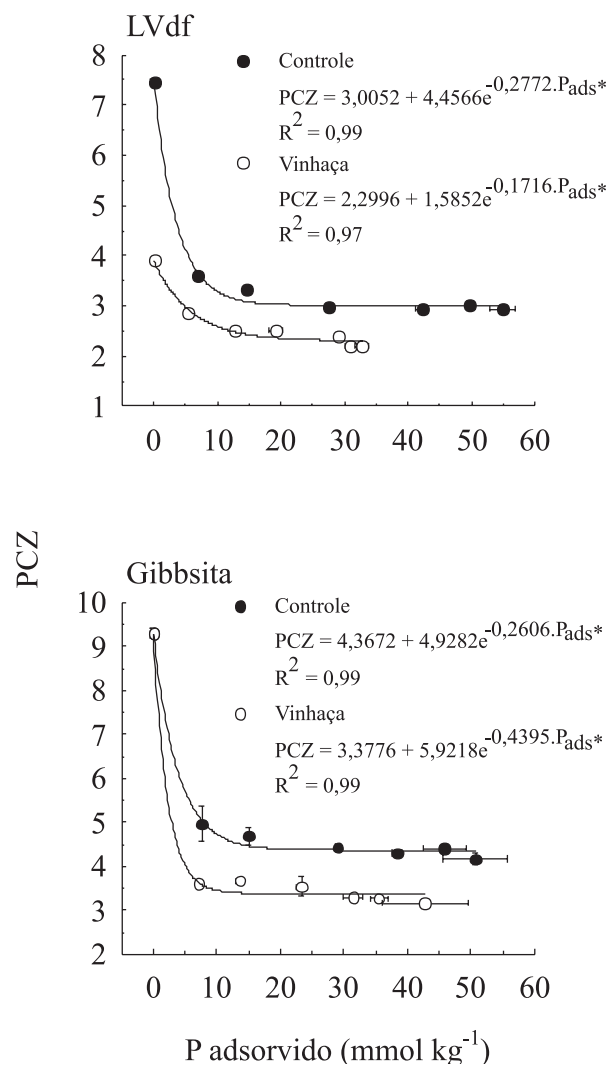

Figura 2. Efeito da quantidade adsorvida de P sobre o ponto de carga zero (PCZ) da fração argila do Latossolo Vermelho Distroférrico (LVdf) e gibbsita. Barras de erro bidirecionais indicam o desvio-padrão da média $(n=3)$. *Significância dos modelos ajustados ( $p<0,0001$ - Teste F)

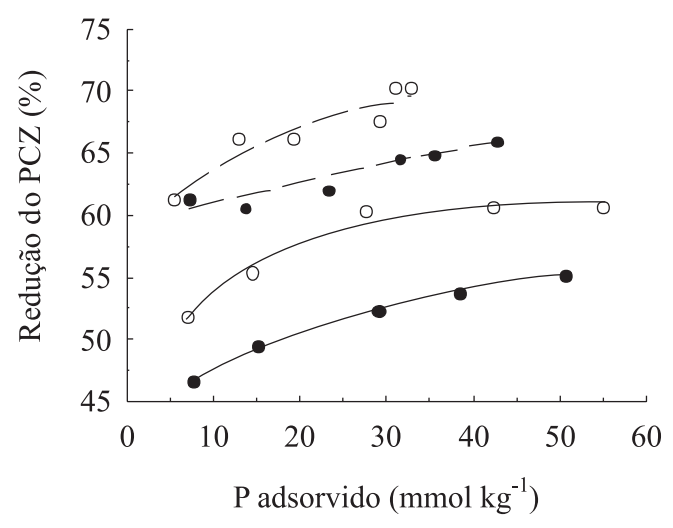

$$
\begin{aligned}
& \text { Gibbsita - controle } \\
& \%=43,2480+13,9952(1-\exp (-0,0370 \cdot \mathrm{Pads})) \mathrm{R}^{2}=0,99(\mathrm{p}<0,01) \\
& \text { - - - } \\
& \text { Gibbsita + vinhaça } \\
& \%=59,2558+0,1540 \cdot \text { Pads } \mathrm{R}^{2}=0,90(\mathrm{p}<0,01) \\
& \frac{0}{\text { LVdf - controle }} \\
& \%=44,2984+16,9842(1-\exp (-0,0812 \cdot \mathrm{Pads})) \mathrm{R}^{2}=0,98(\mathrm{p}<0,05) \\
& -\mathrm{O}- \\
& \text { LVdf + vinhaça } \\
& \%=58,1115+13,9521(1-\exp (-0,0533 \cdot \text { Pads })) R^{2}=0,89(p<0,05)
\end{aligned}
$$

Figura 3. Redução relativa do ponto de carga zero (PCZ) em função da quantidade adsorvida de $P$, na ausência e presença da vinhaça, da fração argila do Latossolo Vermelho Distroférrico (LVdf) e gibbsita 
gura 4). O LVdf e a gibbsita apresentaram uma CMAP de 52 e 49 mmol kg-1 , respectivamente.

Pozza et al. ${ }^{36}$ trabalhando com esse mesmo material gibbsítico deste trabalho e nas mesmas condições, exceto a força iônica da solução inicial de $\mathrm{P}$, que foi três vezes maior, encontraram uma maior CMAP (66 mmol kg-1). Em solos com carga variável e em condição de $\mathrm{pH}$ inferior ao PCZ, como foram conduzidos os ensaios de adsorção, o aumento da concentração eletrolítica leva a um aumento das cargas positivas dos coloides do solo, ${ }^{1}$ o que favorece a adsorção de $\mathrm{P}$, conforme observado por Pardo et al.. ${ }^{44}$

No LVdf, observa-se efeito da vinhaça na redução das quantidades adsorvidas em todas as soluções iniciais de $\mathrm{P}$, reduzindo também a CMAP de 52 para $33 \mathrm{mmol} \mathrm{kg}^{-1}$ (Figura 4). Efeito menos expressivo foi observado para a gibbsita, reduzindo a CMAP de 49 para $41 \mathrm{mmol} \mathrm{kg}^{-1}$.

$\mathrm{O}$ efeito da vinhaça na redução da adsorção de $\mathrm{P}$ pode estar relacionado com as alterações nas cargas superficiais das partículas. Nas condições em que foi realizado o ensaio $(\mathrm{pH}=5,5 ; \mathrm{pH}<\mathrm{PCZ}$
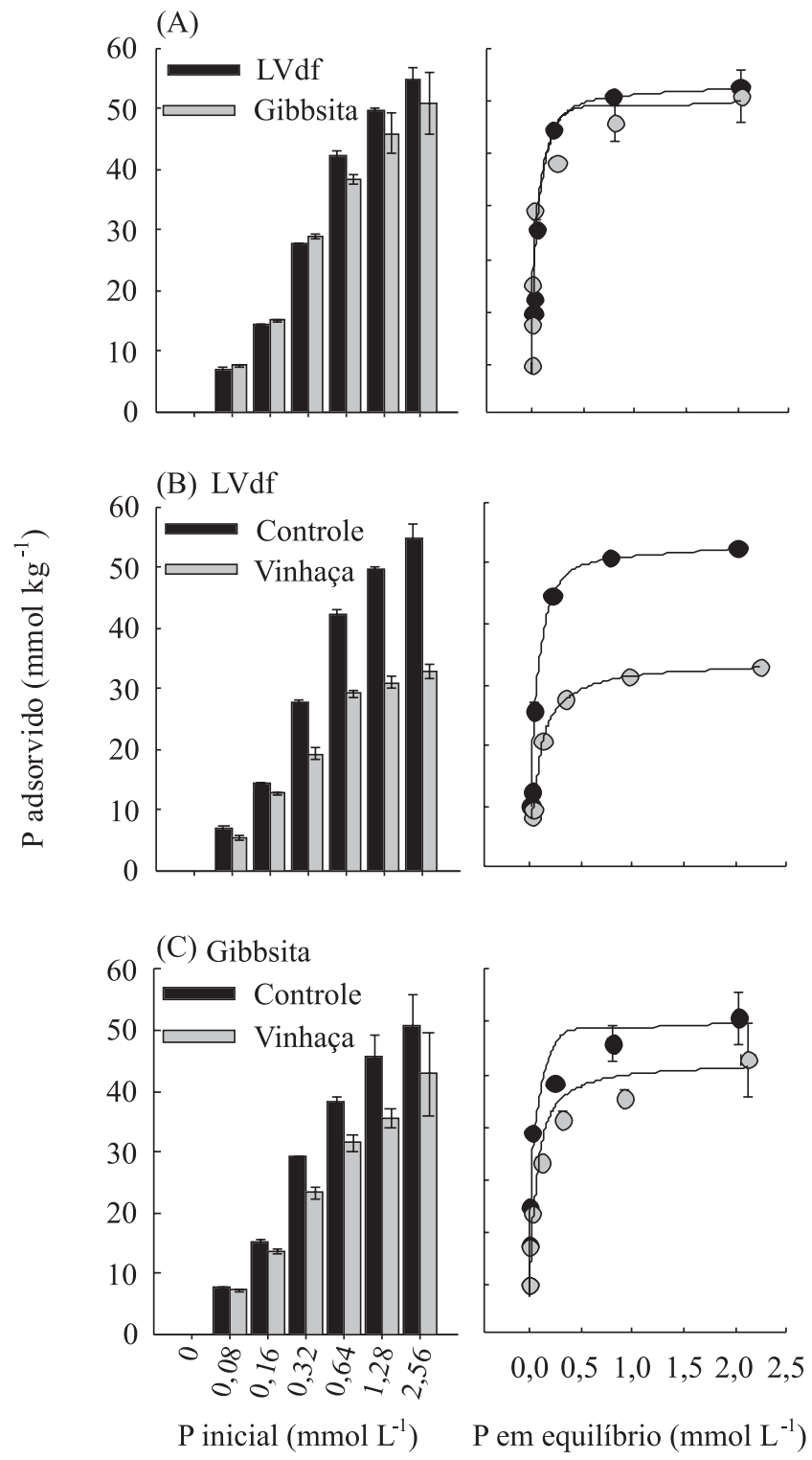

Figura 4. Quantidades adsorvidas e isotermas de adsorção ajustadas ao modelo de Langmuir. (A) Amostras- controle do LVdf e gibbsita. Efeito da vinhaça na adsorção de P na fração argila do $L V d f(B)$ e gibbsita (C). Barras de erro indicam o desvio-padrão da média $(n=3)$ dos materiais), a fração argila do LVdf apresentou um potencial zeta positivo (+10 mV) (Figura 1), ou seja, presença de uma carga líquida positiva. Em condições de $\mathrm{pH} 5,5$, a espécie predominante do fósforo é o $\mathrm{H}_{2} \mathrm{PO}_{4}^{-}$. Dessa forma, a adsorção ocorre pela ligação das cargas negativas do íon fosfato com as cargas positivas. Quando o material foi tratado com vinhaça, observou-se uma redução significativa do potencial zeta, atingindo $-9 \mathrm{mV}$ ( $\mathrm{pH} 5,5)$, havendo uma carga líquida negativa desfavorecendo a adsorção de P. A adsorção de moléculas orgânicas à superfície faz com que seus ligantes se orientem para a solução, ${ }^{45}$ tornando o potencial zeta mais negativo. Para a gibbsita, também se observou uma redução do potencial zeta $(+44$ para +2 $\mathrm{mV}$ ) sem, contudo, haver uma carga líquida negativa, ou seja, em condições de $\mathrm{pH}$ 5,5, a fração argila ainda se manteve positivamente carregada, o que pode ajudar a explicar a redução menos expressiva da adsorção de P (Figura 4).

Com base nos resultados obtidos, infere-se que em solos ricos em óxidos de ferro e de alumínio, como os Latossolos, de ocorrência generalizada no Brasil, a aplicação de vinhaça como fertilizante pode contribuir para o aumento da disponibilidade de $\mathrm{P}$ para as plantas. Além disso, a vinhaça causou importantes alterações nas cargas superficiais dos coloides minerais do solo, como redução do PCZ e geração de cargas negativas. Tais modificações estão relacionadas com fenômenos de superfície, como dispersão/floculação do solo, capacidade de troca de cátions e ânions, adsorção de metais e pesticidas, entre outros.

\section{CONCLUSÕES}

A vinhaça alterou o potencial zeta da fração argila dos solos, tornando a fração argila mais negativamente carregada e reduzindo substancialmente a quantidade de cargas positivas em uma ampla faixa de $\mathrm{pH}$.

A adsorção de $\mathrm{P}$ tornou a superfície mais eletronegativa e reduziu significativamente o PCZ da fração argila dos solos, sendo esses efeitos potencializados na presença de vinhaça.

Em ambos os solos, independentemente do tratamento com vinhaça, o PCZ da fração argila diminuiu exponencialmente com o aumento da quantidade adsorvida de $\mathrm{P}$.

O tratamento da fração argila com vinhaça reduziu a adsorção de $\mathrm{P}$, possivelmente devido ao bloqueio dos sítios de adsorção de $\mathrm{P}$ pelos compostos orgânicos da vinhaça e, também, pelo aumento da carga líquida negativa da fração argila.

\section{MATERIAL SUPLEMENTAR}

Atributos químicos e mineralógicos do LVdf e gibbsita estão apresentados nas Tabelas $1 \mathrm{~S}$ e 2S. Na Tabela 3S são apresentados os modelos polinomiais cúbicos ajustados para a variação do potencial zeta em função do $\mathrm{pH}$, sob efeito da vinhaça e das concentrações iniciais de $\mathrm{P}$, para a fração argila dos materiais de solo utilizados. Os difratogramas de raios-X da fração argila do LVdf e gibbsita estão na Figura 1S. Este material encontra-se disponível em http:// quimicanova.sbq.org.br, na forma de arquivo PDF, com acesso livre.

\section{AGRADECIMENTOS}

À Fundação de Amparo à Pesquisa de Minas Gerais - FAPEMIG, pela aprovação dos recursos financeiros do projeto (Protocolo 2722/06). Ao CNPq pela concessão de bolsas aos autores.

\section{REFERÊNCIAS}

1. Sumner, M. E. Em The electrical double layer and clay dispersion; Sumner, M. E.; Stewart, B. A., eds.; Lewis: Boca Raton, 1992, cap. 2. 
2. Chorom, M.; Rengasamy, P.; Eur. J. Soil Sci. 1995, 46, 657.

3. Gillman, G. P.; Aust. J. Soil Res. 1985, 23, 643.

4. Clausen, L.; Fabricius, I.; Madsen, L.; J. Environ. Qual. 2001, 30, 846.

5. Appel, C.; Ma, L.; J. Environ. Qual. 2002, 31, 581.

6. Tan, K. H.; Principles of soil chemistry, $2^{\text {nd }}$ ed., M. Dekker: New York, 1993.

7. Sparks, D. L.; Environmental soil chemistry, Academic: California, 2003.

8. Fontes, M. P. F.; Camargo, O. A.; Sposito, A.; Scientia Agrícola 2001, 58,627 .

9. Weber, O. L. S.; Chitolina, J. C.; Camargo, O. A.; Alleoni, L. R. F.; R. Bras. Ci. Solo 2005, 29, 867.

10. Aydin, M.; Yano, T.; Kilic, S.; Soil Sci. Soc. Am. J. 2004, 68, 450.

11. Alkan, M.; Demirbas, Ö.; Do an, M.; Microporous Mesoporous Mater. 2005, 83, 51 .

12. Ranst, E. van; Shamshuddin, J.; Baert, G.; Dzwowa, P. K.; Eur. J. Soil Sci. 1998, 49, 243.

13. Zhuang, J.; Yu, G. R.; Chemosphere 2002, 49, 619.

14. Anda, M.; Shamshuddin, J.; Fauziah, C. I.; Omar, S. R. S.; Geoderma 2008, 143, 153 .

15. Stoop, W. A.; Geoderma 1980, 23, 303.

16. Lima, J. M.; Anderson, S. J.; Curi, N.; Soil Sci. Soc. Am. J. 2000, 64, 892.

17. Marchi, G.; Guilherme, L. R. G.; Chang, A. C.; Curi, N.; Guerreiro, M. C.; Commun. Soil Sci. Plant Anal. 2006, 37, 1357.

18. Resende, M.; Curi, N.; Rezende, S. B.; Corrêa, G. F.; Pedologia: base para distinção de ambientes, UFLA: Lavras, 2007.

19. Eberhardt, D. N.; Vendrame, P. R. S.; Becquer, T.; Guimarães, M. F.; $R$. Bras. Ci. Solo 2008, 32, 1009.

20. Hingston, F. J.; Posner, A. M.; Quirk, J. P.; J. Soil Sci. 1974, 25, 16.

21. Giaveno, C.; Celi, L.; Cessa, R. M. A.; Prati, M.; Bonifacio, E.; Barberis, E.; Soil Sci. 2008, 173, 694.

22. Nóbrega, J. C. A.; Lima, J. M.; Guerreiro, M. C.; Regitano, R. L. O.; Moreno, S. A. C.; Pesq. Agropec. Bras. 2005, 40, 503.

23. Pierangeli, M. A. P.; Nóbrega, J. C. A.; Lima, J. M.; Guilherme, L. R. G.; Arantes, S. A.C. M.; Agrária 2009, 4, 42.

24. Andrade, F. V.; Mendonça, E. S.; Alvarez V. H.; Novais, R. F.; R. Bras. Ci. Solo 2003, 27, 1003.
25. Siddique, M. T.; Robinson, J. S.; J. Environ. Qual. 2003, 32, 1114.

26. Negassa, W.; Dultz, S.; Schlichting, A.; Leinweber, P.; Soil Sci. 2008, $173,587$.

27. Glória, N. A.; Orlando Filho, J.; Aplicação de vinhaça como fertilizante, COOPERSUCAR: São Paulo, 1983.

28. Leal, J. R.; Amaral Sobrinho, N. M. B.; Velloso, A. C. X.; Rossiello, R. O. P.; R. Bras. Ci. Solo 1983, 7, 257.

29. Neves, M. C. P.; Lima, L. T.; Dobereiner, J.; R. Bras. Ci. Solo 1983, 7, 131.

30. Mattiazo, M. E.; Glória, N. A.; Water Sci. Technol. 1987, 19, 1293.

31. Sengik, E.; Ribeiro, A. C.; Condé, A. R.; R. Bras. Ci. Solo 1988, 12, 11.

32. Silva, A. J. N.; Ribeiro, M. R.; R. Bras. Ci. Solo 1998, 22, 291.

33. Doelsch, E.; Masion, A.; Cazevieille, P.; Condom, N.; Waste Management 2009, 29, 1929.

34. Benke, M. B.; Mermut, A. R.; Shariatmadari, H.; Geoderma 1999, 91, 47.

35. Inda Júnior, A. V.; Bayer, C.; Conceição, P. C.; Boeni, M.; Salton, J. C.; Tonin, A. T.; Ciência Rural 2007, 37, 1301.

36. Pozza, A. A. A.; Curi, N.; Guilherme, L. R. G.; Marques, J. J. G. S. M.; Costa, E. T. S.; Zuliani, D. Q.; Motta, P. E. F.; Martins, R. S.; Oliveira, L. C. A.; Quim. Nova 2009, 32, 99.

37. Empresa Brasileira de Pesquisa Agropecuária; Manual de métodos de análises de solo, $2^{\mathrm{a}}$ ed., CNPS: Rio de Janeiro, 1997.

38. Walkley, A.; Black, I. A.; Soil Sci. 1934, 37, 29.

39. Bolster, C. H.; Hornberger, G. M.; Soil Sci. Soc. Am. J. 2007, 71, 1796.

40. Scientific Graphing Software; SigmaPlot 10.0, Jandel: San Rafael, 2006.

41. Hou, T.; Xu, R.; Zhao, A.; Colloids Surf., A 2007, 297, 91.

42. Pozza, A. A. A.; Curi, N.; Costa, E. T. S.; Guilherme, L. R. G.; Marques, J. J. G. S. M.; Motta, P. E. F.; Pesq. Agropec. Bras. 2007, 42, 1627.

43. Dobbss, L. B.; Canellas, L. P.; Alleoni, L. R. F.; Rezende, C. E.; Fontes, M. P. F.; Velloso, A. C. X.; R. Bras. Ci. Solo 2008, 32, 985.

44. Pardo, M. T.; Guadalix, M. E.; Garcia-Gonzalez, M. T.; Geoderma 1992, $54,275$.

45. Rahnemaie, R.; Hiemstra, T.; Riemsdijk, W. H.; J. Colloid Interface Sci. 2006, 293, 312 . 


\section{CARGAS SUPERFICIAIS DA FRAÇÃO ARGILA DE SOLOS INFLUENCIADAS PELA VINHAÇA E FÓSFORO}

\section{Bruno Teixeira Ribeiro*}

Instituto Federal de Educação, Ciência e Tecnologia do Sul de Minas Gerais, Campus Machado, 37750-000 Machado - MG, Brasil José Maria de Lima, Nilton Curi, Geraldo César de Oliveira e Pedro Luiz Terra Lima

Departamento de Ciência do Solo, Universidade Federal de Lavras, CP 37, 37200-000 Lavras - MG, Brasil

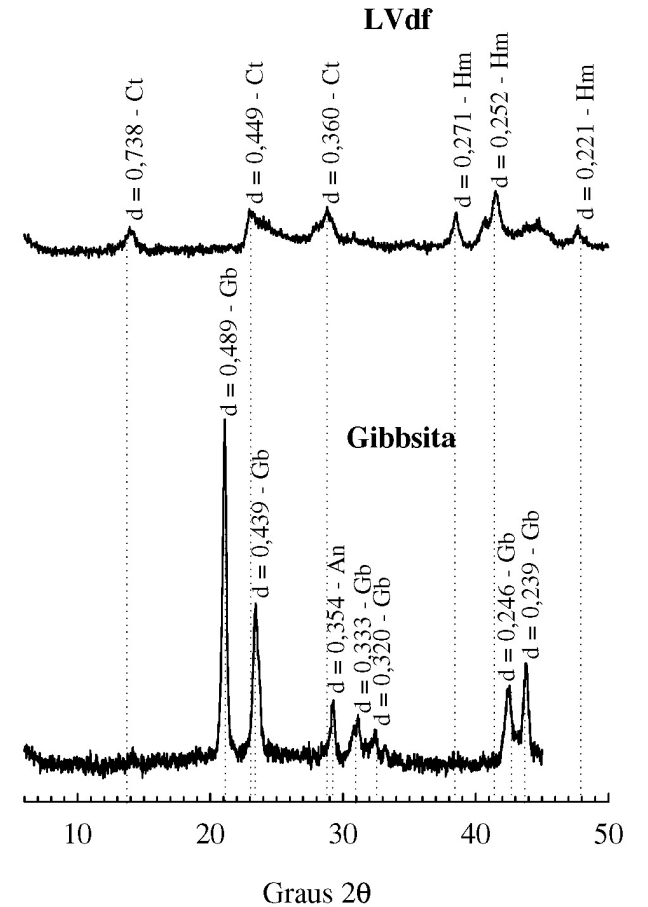

Figura 1S. Difratogramas de raios-X da fração argila do Latossolo Vermelho Distroférrico (LVdf) e da gibbsita. Ct: caulinita; Gb: gibbsita; An:anatásio; Hm: Hematita

Tabela 1S. Teor de óxidos, índices de intemperismo e teor de carbono orgânico de Latossolo Vermelho Distroférrico (LVdf) e gibbsita

\begin{tabular}{lcccccc}
\hline & $\mathrm{SiO}_{2}{ }^{11}$ & $\mathrm{Al}_{2} \mathrm{O}_{3}{ }^{21}$ & $\mathrm{Fe}_{2} \mathrm{O}_{3}{ }^{2 /}$ & $\mathrm{Ki}$ & $\mathrm{Kr}$ & $\begin{array}{c}\mathrm{C}_{\text {org. }} \\
-\mathrm{g} \mathrm{kg}^{-1}-\end{array}$ \\
\hline LVdf & $--------\mathrm{g} \mathrm{kg}^{-1}---------$ & & & 6,0 \\
gibbsita & 864 & 256 & 177 & 1,88 & 1,31 & 6,30 \\
\hline
\end{tabular}

${ }^{1 /}$ Obtido após ataque alcalino e determinado por gravimetria; ${ }^{2 / O ́ x i d o s ~ o b-~}$ tidos pelo ataque sulfúrico; Ki: relação molecular $\left(\mathrm{SiO}_{2} / \mathrm{Al}_{2} \mathrm{O}_{3}\right)$; Kr: relação molecular $\left(\mathrm{SiO}_{2} / \mathrm{Al}_{2} \mathrm{O}_{3}+\mathrm{Fe}_{2} \mathrm{O}_{3}\right) ; \mathrm{C}_{\text {org }}$ : carbono orgânico, Embrapa ${ }^{37}$

Tabela 2S. Caracterização do complexo sortivo do Latossolo Vermelho Distroférrico (LVdf) e da gibbsita

\begin{tabular}{lcccccccccc}
\hline & $\mathrm{pH}$ & $\mathrm{P}$ & $\mathrm{K}$ & $\mathrm{Ca}$ & $\mathrm{Mg}$ & $\mathrm{Al}$ & $\mathrm{H}+\mathrm{Al}$ & $\mathrm{SB}$ & $\mathrm{T}$ & $\mathrm{V}$ \\
& & $---\mathrm{mg} \mathrm{dm}^{-3}---$ & $-----------\mathrm{mmol}_{\mathrm{c}} \mathrm{dm}^{-3}$ & --------- & $\%$ \\
\hline LVdf & 5,3 & 2,0 & 28,0 & 23,0 & 14,0 & 10,0 & 63,0 & 37,7 & 10,0 & 37,0 \\
gibbsita & 5,5 & 1,2 & 6,0 & 1,0 & 1,0 & 1,0 & 23,0 & 2,2 & 25,0 & 87,0 \\
\hline
\end{tabular}

pH em $\mathrm{H}_{2} \mathrm{O}$ (relação solo: água 1:2,5); $\mathrm{SB}$ : soma de bases; T: capacidade de troca de cátions potencial a pH 7,0; V: saturação por bases, Embrapa ${ }^{37}$
Tabela 3S. Modelos polinomiais cúbicos $\left(\mathrm{y}=\mathrm{y}_{0}+\mathrm{ax}+\mathrm{bx}^{2}+\right.$ $\left.\mathrm{cx}^{3}\right)$ ajustados para a variação do potencial zeta $(\zeta)$ em função do $\mathrm{pH}$, sob efeito da vinhaça e das concentrações iniciais de P, para a fração argila do Latossolo Vermelho Distroférrico (LVdf) e gibbsita

\begin{tabular}{|c|c|c|c|c|}
\hline \multirow{3}{*}{\multicolumn{2}{|c|}{$\begin{array}{l}\text { Tratamento } \\
\text { P (mM) Vinhaça }\end{array}$}} & \multirow{3}{*}{$\begin{array}{c}\text { Modelo ajustado* } \\
\text { LVdf } \\
\end{array}$} & \multirow[t]{3}{*}{$\mathrm{R}^{2}$} & \multirow[t]{3}{*}{$\mathrm{R}_{\text {ajustado }}^{2}$} \\
\hline & & & & \\
\hline & & & & \\
\hline \multirow[t]{2}{*}{0,00} & sem & $\zeta=15,99+3,01 \mathrm{pH}-0,94 \mathrm{pH}^{2}+0,03 \mathrm{pH}^{3}$ & 0,96 & 0,95 \\
\hline & com & $\zeta=48,25-19,03 \mathrm{pH}+2,00 \mathrm{pH}^{2}-0,07 \mathrm{pH}^{3}$ & 0,99 & 0,99 \\
\hline \multirow[t]{2}{*}{0,08} & sem & $\zeta=40,47-19,16 \mathrm{pH}+2,70 \mathrm{pH}^{2}-0,14 \mathrm{pH}^{3}$ & 0,97 & 0,97 \\
\hline & com & $\zeta=33,60-15,94 \mathrm{pH}+1,68 \mathrm{pH}^{2}-0,07 \mathrm{pH}^{3}$ & 0,97 & 0,97 \\
\hline \multirow[t]{2}{*}{0,16} & sem & $\zeta=51,41-24,34 \mathrm{pH}+3,18 \mathrm{pH}^{2}-0,14 \mathrm{pH}^{3}$ & 0,99 & 0,98 \\
\hline & com & $\zeta=37,23-20,46 \mathrm{pH}+2,57 \mathrm{pH}^{2}-0,12 \mathrm{pH}^{3}$ & 0,99 & 0,99 \\
\hline \multirow[t]{2}{*}{0,32} & sem & $\zeta=61,98-32,88 \mathrm{pH}+4,77 \mathrm{pH}^{2}-0,24 \mathrm{pH}^{3}$ & 0,97 & 0,97 \\
\hline & com & $\zeta=38,02-21,07 \mathrm{pH}+2,63 \mathrm{pH}^{2}-0,12 \mathrm{pH}^{3}$ & 0,99 & 0,99 \\
\hline \multirow[t]{2}{*}{0,64} & sem & $\zeta=52,21-27,25 \mathrm{pH}+3,77 \mathrm{pH}^{2}-0,18 \mathrm{pH}^{3}$ & 0,99 & 0,98 \\
\hline & com & $\zeta=33,65-18,90 \mathrm{pH}+2,22 \mathrm{pH}^{2}-0,10 \mathrm{pH}^{3}$ & 0,99 & 0,99 \\
\hline \multirow[t]{2}{*}{1,28} & sem & $\zeta=35,85-16,62 \mathrm{pH}+1,77 \mathrm{pH}^{2}-0,07 \mathrm{pH}^{3}$ & 0,98 & 0,98 \\
\hline & com & $\zeta=31,57-18,71 \mathrm{pH}+2,20 \mathrm{pH}^{2}-0,09 \mathrm{pH}^{3}$ & 0,99 & 0,99 \\
\hline \multirow[t]{3}{*}{2,56} & sem & $\zeta=36,02-17,41 \mathrm{pH}+1,99 \mathrm{pH}^{2}-0,09 \mathrm{pH}^{3}$ & 0,98 & 0,98 \\
\hline & com & $\zeta=37,38-22,69 \mathrm{pH}+2,85 \mathrm{pH}^{2}-0,13 \mathrm{pH}^{3}$ & 0,99 & 0,99 \\
\hline & & gibbsita & & \\
\hline \multirow[t]{2}{*}{0,00} & sem & $\zeta=32,97+18,09 \mathrm{pH}-3,77 \mathrm{pH}^{2}+0,15 \mathrm{pH}^{3}$ & 0,98 & 0,97 \\
\hline & com & $\zeta=20,95-4,86 \mathrm{pH}+0,94 \mathrm{pH}^{2}-0,07 \mathrm{pH}^{3}$ & 0,95 & 0,94 \\
\hline \multirow[t]{2}{*}{0,08} & sem & $\zeta=57,61-20,42 \mathrm{pH}+2,27 \mathrm{pH}^{2}-0,10 \mathrm{pH}^{3}$ & 0,97 & 0,97 \\
\hline & com & $\zeta=42,12-16,38 \mathrm{pH}+1,47 \mathrm{pH}^{2}-0,05 \mathrm{pH}^{3}$ & 0,98 & 0,98 \\
\hline \multirow[t]{2}{*}{0,16} & sem & $\zeta=54,37-18,42 \mathrm{pH}+1,77 \mathrm{pH}^{2}-0,06 \mathrm{pH}^{3}$ & 0,99 & 0,99 \\
\hline & com & $\zeta=19,93-3,21 \mathrm{pH}-0,83 \mathrm{pH}^{2}+0,06 \mathrm{pH}^{3}$ & 0,99 & 0,99 \\
\hline \multirow[t]{2}{*}{0,32} & sem & $\zeta=51,34-17,18 \mathrm{pH}+1,45 \mathrm{pH}^{2}-0,04 \mathrm{pH}^{3}$ & 0,99 & 0,99 \\
\hline & com & $\zeta=25,32-6,24 \mathrm{pH}-0,40 \mathrm{pH}^{2}+0,04 \mathrm{pH}^{3}$ & 0,98 & 0,98 \\
\hline \multirow[t]{2}{*}{0,64} & sem & $\zeta=52,38-16,96 \mathrm{pH}+1,23 \mathrm{pH}^{2}-0,03 \mathrm{pH}^{3}$ & 0,99 & 0,99 \\
\hline & com & $\zeta=30,92-10,88 \mathrm{pH}+0,49 \mathrm{pH}^{2}-0,01 \mathrm{pH}^{3}$ & 0,99 & 0,99 \\
\hline \multirow[t]{2}{*}{1,28} & sem & $\zeta=46,11-12,76 \mathrm{pH}+0,51 \mathrm{pH}^{2}-0,0028 \mathrm{pH}^{3}$ & 0,99 & 0,99 \\
\hline & com & $\zeta=23,10-7,04 \mathrm{pH}-0,11 \mathrm{pH}^{2}+0,02 \mathrm{pH}^{3}$ & 0,99 & 0,98 \\
\hline \multirow[t]{2}{*}{2,56} & sem & $\zeta=46,26-14,29 \mathrm{pH}+0,82 \mathrm{pH}^{2}-0,01 \mathrm{pH}^{3}$ & 0,99 & 0,98 \\
\hline & com & $\zeta=13,43-1,39 \mathrm{pH}-1,18 \mathrm{pH}^{2}+0,08 \mathrm{pH}^{3}$ & 0,99 & 0,98 \\
\hline
\end{tabular}

${ }^{*} \mathrm{P}<0,0001$ (Teste F).

*e-mail: ribeiro.bt@gmail.com 